\title{
On Finite-Time Feedback Control for Switched Discrete-Time Systems under Fast and Slow Switching
}

\author{
Liaoliang Xiao' ${ }^{1}$ and Mingyang $\mathbf{Y u}^{2}$ \\ ${ }^{1}$ Hunan Railway Professional Technology College, Zhuzhou, Hunan 412001, China \\ ${ }^{2}$ School of Information Science and Engineering, Central South University, Changsha 410075, China \\ Correspondence should be addressed to Liaoliang Xiao; llxiaohn@126.com
}

Received 4 October 2014; Accepted 10 November 2014; Published 24 December 2014

Academic Editor: Muhammad Naveed Iqbal

Copyright (c) 2014 L. Xiao and M. Yu. This is an open access article distributed under the Creative Commons Attribution License, which permits unrestricted use, distribution, and reproduction in any medium, provided the original work is properly cited.

\begin{abstract}
The problem of finite-time stabilization for switched discrete-time systems under both fast and slow switching is addressed. In the fast switching case, the designed static state feedback controller combines controllers for each subsystem and resetting controller at switching instant, it is shown that the resetting controller can reduce the conservativeness on controller design. Then the results are extended to output feedback controller design. Under slow switching, both static state feedback and output feedback controller are designed with admissible average dwell time, respectively. Several numerical examples are given to illustrate the proposed results within this paper.
\end{abstract}

\section{Introduction}

A switched system consists of a finite number of subsystems that are switched according to the time variation of the system's mode of operation. Many physical processes possess switched and hybrid nature [1-3], and switched systems arise in many engineering applications, for example, in motor engine control [4], constrained robotics [5], and networked control systems [6]. Due to their ubiquity in modern engineering problems, switched systems are receiving increasing interest and attention as the recent books $[1,2]$ and survey articles $[3,7,8]$ indicate. Stability and stabilization are the main concerns in the field of switched systems. Many Lyapunov function techniques are effective tools dealing with switched systems [9-11]. Dwell time and average dwell time approaches were employed to study the stability and stabilization of time-dependent switched systems [12-14].

On the other hand, the finite-time stability is a concept dealing with the boundness of system during a fixed finitetime interval, which mainly focuses on admitting the state does not exceed a certain bound during a fixed finite-time interval, for instance, to avoid saturations or the excitation of nonlinear dynamics. A distinction should be made between classical Lyapunov stability and finite-time stability (or shorttime stability). The concept of Lyapunov asymptotic stability is largely known to the control community; conversely a system is said to be finite-time stable if, once we fix a time interval, its state does not exceed some bounds during this time interval. Often asymptotic stability is enough for practical applications, but there are some cases where large values of the state are not acceptable, for instance, in the presence of saturations. In these cases, we need to check that these unacceptable values are not attained by the state; for these purposes finite-time stability could be used. Some early results about finite-time stability can be found in [15-17]. More recently, [18] investigated the output feedback finitetime stabilization for continuous linear system. Finite-time stability and stabilization for discrete linear system were investigated in [19]. Moreover, the robust finite-time control for linear switched discrete-time system with norm-bounded disturbance was considered in [20], and continuous-time case is considered in [21].

Similar as indicated for asymptotic stability, the finitetime stability is supposed to be affected significantly by switching among several subsystems $[20,21]$. Thus, besides Lyapunov asymptotic stability, another important system property we are interested in is the boundness of the state during the short time interval in which the switching occurs, and it is explicit that the boundness of state during the short time interval could be influenced significantly by the switching. 
To avoid the state reaching the unacceptable large values caused by switching during the short time interval, the boundness property of state, that is, the finite-time stability, needs to be considered when we design the controller and switching law. However, most of the existing stability results were about Lyapunov stability; few results have been reported in literature about the finite-time stability of switched systems so far. This motivates the present study.

In this paper, solutions for finding state and output feedback controller guaranteeing the finite-time stabilization of switched discrete-time system are given in both fast switching and slow switching case. In fast switching case, a natural idea is to find a single common positive matrix satisfying finite-time stabilization condition for each subsystem to ensure the finite-time stability of closed-loop switched system which is similar to the common Lyapunov function approach dealing with asymptotic stability. However, it often yields overly conservativeness. To reduce the conservativeness our approach is based on searching for a set of positive matrices; furthermore it is worth noting that the controller combines the controller for each subsystem and resetting controller at switching instant which generates a proper state impulsive jumping which could further reduce the conservativeness in finite-time controller design. In slow switching case, the controller design is proposed including the controllers for subsystems and the admissible average dwell time. The results in fast and slow switching case are both extended to output feedback case. Several numerical simulations are proposed to show the effectiveness of our approach.

The rest of this paper is organized as follows. In Section 2 the problem formulation and some preliminaries are introduced. The main results, finite-time stabilization under fast switching and slow switching, are given in Sections 3 and 4 , respectively; several numerical simulations are given to illustrate our proposed results. Conclusions are given in Section 5 .

Notations. The notations used in this paper are fairly standard. The superscript " $T$ " stands for matrix transposition, $\mathbb{R}^{n}$ denotes the $n$-dimensional Euclidean space and $\mathbb{Z}^{+}$represents the set of nonnegative integers, and the notation $\|$. $\|$ refers to the Euclidean norm. In addition, in symmetric block matrices, we use $*$ as an ellipsis for the terms that are introduced by symmetry and $\operatorname{diag}\{\cdots\}$ stands for a blockdiagonal matrix. $\lambda_{\min }(P)$ and $\lambda_{\max }(P)$ stand for the smallest and the largest eigenvalue of matrix $P$. The notation $P>0$ $(P \geq 0)$ means $P$ is real symmetric and positive definite (semipositive definite).

\section{Preliminaries}

In this paper, a switched discrete-time system we consider is described as follows:

$$
\begin{gathered}
x(k+1)=A_{i(k)} x(k)+B_{i(k)} u(k), \quad x(0)=x_{0}, \\
y(k)=C_{i(k)} x(k),
\end{gathered}
$$

where $x(k) \in \mathbb{R}^{n}$ is the discrete state, $u(k) \in \mathbb{R}^{m}$ is the control input, and $y(k) \in \mathbb{R}^{q}$ is the measurement of the system. $A_{i}, B_{i}$, and $C_{i}$ are real known constant matrices with appropriate dimensions. $i(k): \mathbb{Z}^{+} \rightarrow \mathscr{I}=\{1,2, \ldots, N\}$ is a piecewisely constant function of discrete-time $k$, called switching law or switching signal, which takes its values in finite set $\mathscr{I}$. $N>0$ is the number of subsystems. For simplicity, at any arbitrary discrete-time $k \in \mathbb{Z}^{+}$the switching signal $i(k)$ is denoted by $i$. Given switched system ((1a), (1b)), the switching sequence can be defined as $\mathcal{S}:=$ $\left\{k_{0}, k_{1}, k_{2}, \ldots, k_{l}, \ldots\right\}$, where $k_{0}$ denotes the initial time and $k_{l}$ denotes the $l$ th switching instant. In this paper, we assume that the switching signal is available in real time; that is, the activated subsystem is explicitly known at each switching instant and the corresponding controller can be activated immediately.

For switched discrete-time system, the general conception of finite-time stability concerns the boundness of discrete state $x(k)$ over finite discrete-time interval $[0, M], M \in \mathbb{Z}^{+}$ with respect to given initial condition $x_{0}$. This conception can be formulized through following definition.

Definition 1 (finite-time stability). Switched system

$$
x(k+1)=A_{i} x(k), \quad x(0)=x_{0},
$$

is said to be finite-time stable with respect to $(\delta, \varepsilon, R, M)$, where $0 \leq \delta<\varepsilon, R$ is a positive definite matrix, and $M \in \mathbb{Z}^{+}$, if $x^{T}(k) R x(k)<\varepsilon^{2}, \forall k \in\{1,2, \ldots, M\}$ whenever $x_{0}^{T} R x_{0}<\delta^{2}$.

Then we recall the following lemma, which will be used in the proof of our main results.

Lemma 2 (see [22]). The linear matrix inequality

$$
S=\left[\begin{array}{ll}
S_{11} & S_{12} \\
S_{12}^{T} & S_{22}
\end{array}\right]<0,
$$

where $S_{11}=S_{11}^{T}$ and $S_{22}=S_{22}^{T}$ are equivalent to

$$
S_{22}<0, \quad S_{11}-S_{12} S_{22}^{-1} S_{12}^{T}<0 .
$$

Lemma 2 is the well-known Schur complement lemma.

\section{Finite-Time Stabilization under Fast Switching}

In this section we consider the finite-time stabilization for switched discrete-time system under fast switching. A set of static state feedback controllers are given as $u(k)=K_{i} x(k)$, $\forall k \in \mathbb{Z}^{+} \backslash \mathcal{S}$ during the subsystems activation time and $u(k)=$ $K_{i, j} x(k), \forall k \in \mathcal{S}$ and $i \neq j,(i, j) \in \mathscr{I} \times \mathscr{I}$ at the switching instant when system ((1a), (1b)) switches from subsystem $i$ to $j$. Then the closed-loop switched system becomes

$$
x(k+1)=\left(A_{i}+B_{i} K_{i}\right) x(k), \quad x(0)=x_{0}, \forall k \in \mathbb{Z}^{+} \backslash \mathcal{S},
$$

$$
x(k+1)=\left(A_{i}+B_{i} K_{i, j}\right) x(k), \quad \forall k \in \mathcal{S} .
$$


Remark 3. From (5a) and (5b) we see that the state impulsive jumping idea is applied since the state feedback controller combines controllers for each subsystem and resetting controller generating a proper state impulsive jumping at switching instant.

Before deriving the conditions for finite-time stabilization of switched system ((1a), (1b)), some explicit facts are recalled. For a symmetric positive definite matrix $R \in \mathbb{R}^{n \times n}$, it is easy to verify that $R$ can be factorized according to $R=\left(R^{1 / 2}\right)^{T} R^{1 / 2}$, where $R^{1 / 2} \in \mathbb{R}^{n \times n}$ is a symmetric positive definite matrix. And for any positive definite matrix $R \in \mathbb{R}^{n \times n}$, there always exist $R^{-1} \in \mathbb{R}^{n \times n}$ which is positive definite. We present our first result on controller design ensuring the closed-loop system ((5a), (5b)) finite-time stable by following theorem.

Theorem 4. The closed-loop switched system $((5 a),(5 b))$ is finite-time stable with respect to $(\delta, \varepsilon, R, M)$, if there exist a set of matrices $S_{i}, i \in \mathscr{I}, X_{i, j},(i, j) \in \mathscr{I} \times \mathscr{I}$, and positive scalars $\alpha>0, \beta>0$, and $\gamma \geq 1$ such that the following conditions are satisfied $\forall(i, j) \in \mathscr{I} \times \mathscr{I}$ :

$$
\begin{gathered}
{\left[\begin{array}{cc}
-S_{j} & A_{i} S_{i}+B_{i} X_{i, j} \\
* & -\gamma S_{i}
\end{array}\right]<0,} \\
{\left[\begin{array}{cc}
-S_{i} & \alpha S_{i} R \\
* & -\alpha R
\end{array}\right]<0, \quad\left[\begin{array}{cc}
-\beta R & I \\
* & -S_{i}
\end{array}\right]<0,} \\
\beta \gamma^{M} \delta^{2}-\alpha \varepsilon^{2}<0 .
\end{gathered}
$$

Then the set of state feedback controllers is given by $K_{i}=X_{i, i} S_{i}^{-1}$ and $K_{i, j}=X_{i, j} S_{i}^{-1}$.

Proof. Let $K_{i, i}=K_{i}$ and Substituting $X_{i, j}=K_{i, j} S_{i}, \forall(i, j) \in$ $\mathscr{I} \times \mathscr{I}$ into (6a); we get

$$
\left[\begin{array}{cc}
-S_{j} & A_{i} S_{i}+B_{i} K_{i, j} S_{i} \\
* & -\gamma S_{i}
\end{array}\right]<0
$$

For pre- and postmultiplying (7) by the symmetric matrix $\left[\begin{array}{cc}S_{j}^{-1} & 0 \\ * & S_{i}^{-1}\end{array}\right]$, the following equivalent condition of (7) is obtained:

$$
\left[\begin{array}{cc}
-S_{j}^{-1} & S_{j}^{-1}\left(A_{i}+B_{i} K_{i, j}\right) \\
* & -\gamma S_{i}^{-1}
\end{array}\right]<0 .
$$

Let $P_{j}=S_{j}^{-1}$ and $P_{i}=S_{i}^{-1}$ in (8); we have

$$
\left[\begin{array}{cc}
-P_{j} & P_{j}\left(A_{i}+B_{i} K_{i, j}\right) \\
* & -\gamma P_{i}
\end{array}\right]<0 .
$$

Then by Lemma 2 we get $\forall(i, j) \in \mathscr{I} \times \mathscr{I}$

$$
\begin{aligned}
& {\left[\begin{array}{cc}
-P_{j} & P_{j}\left(A_{i}+B_{i} K_{i, j}\right) \\
* & -\gamma P_{i}
\end{array}\right]<0} \\
& \Longrightarrow\left(A_{i}+B_{i} K_{i, j}\right)^{T} P_{j}\left(A_{i}+B_{i} K_{i, j}\right)-\gamma P_{i}<0 .
\end{aligned}
$$

Let $V_{i}(k)=x^{T}(k) P_{i} x(k), i \in \mathscr{I}$, for each subsystem of closedloop ((5a), (5b)); then we construct following Lyapunov-like function:

$$
V(k)=\sum_{i \in \mathscr{I}} \theta_{i}(k) V_{i}(k)=\sum_{i \in \mathscr{I}} x^{T}(k) \theta_{i}(k) P_{i} x(k),
$$

where $\theta_{i}(\cdot): \mathbb{Z}^{+} \rightarrow\{0,1\}$ and $\sum_{i \in \mathscr{I}} \theta_{i}(k)=1$ is the indication function indicating the activated subsystem. In (14), the case $i=j$ shows that the closed-loop system $((5 a),(5 b))$ works in the $i$ th mode described by $A_{i}$, and the case $i \neq j$ implies the closed-loop system $((5 a),(5 b))$ is switching from subsystem $i$ to $j$ at switching instant $k, k \in\{1,2, \ldots, M\}$. Thus, (10) implies that

$$
V(k+1)<\gamma V(k)
$$

Applying iteratively (12), we obtain

$$
V(k)<\gamma^{k} V(0), \quad \forall k \in\{1,2, \ldots, M\} .
$$

From (11) we know for $\forall i \in \mathscr{I}$ and $\forall k \in\{1,2, \ldots, M\}$

$$
\begin{aligned}
V(k) & =x^{T}(k) P_{i} x(k) \\
& =x^{T}(k) R^{1 / 2} R^{-1 / 2} P_{i} R^{-1 / 2} R^{1 / 2} P_{i} x(k) \\
& =x^{T}(k) R^{1 / 2} Q_{i} R^{1 / 2} x(k) \\
& \geq \inf _{i \in \mathscr{I}}\left\{\lambda_{\min }\left(Q_{i}\right)\right\} x^{T}(k) R x(k),
\end{aligned}
$$

where $Q_{i}=R^{-1 / 2} P_{i} R^{-1 / 2}, i \in \mathscr{I}$.

On the other hand $\forall i \in \mathscr{I}$ we have

$$
\begin{aligned}
\gamma^{k} V(0) & =\gamma^{k} x^{T}(0) P_{i} x(0) \\
& =\gamma^{k} x^{T}(0) R^{1 / 2} Q_{i} R^{1 / 2} x(0) \\
& \leq \gamma^{k} \sup _{i \in \mathscr{I}}\left\{\lambda_{\max }\left(Q_{i}\right)\right\} x^{T}(0) R x(0) ;
\end{aligned}
$$

using the fact $\gamma \geq 1$ and $x^{T}(0) R x(0) \leq \delta^{2}$ we get

$$
\gamma^{k} V(0) \leq \gamma^{M} \sup _{i \in \mathscr{I}}\left\{\lambda_{\max }\left(Q_{i}\right)\right\} \delta^{2} .
$$

Using (13), (14), and (16) altogether the following inequality can be derived:

$$
\begin{array}{r}
x^{T}(k) R x(k) \leq \gamma^{M} \delta^{2} \frac{\sup _{i \in \mathscr{I}}\left\{\lambda_{\text {max }}\left(Q_{i}\right)\right\}}{\inf _{i \in \mathscr{I}}\left\{\lambda_{\text {min }}\left(Q_{i}\right)\right\}}, \\
\forall k \in\{1,2, \ldots, M\} .
\end{array}
$$

Now, to ensure the finite-time stability of closed-loop switched system ((5a), (5b)) with respect to $(\delta, \varepsilon, R, M)$, the following condition should be satisfied:

$$
\frac{\sup _{i \in \mathscr{I}}\left\{\lambda_{\text {max }}\left(Q_{i}\right)\right\}}{\inf _{i \in \mathscr{I}}\left\{\lambda_{\text {min }}\left(Q_{i}\right)\right\}}<\frac{\varepsilon^{2}}{\gamma^{M} \delta^{2}} .
$$


From (6b) and by Lemma 2, we have

$$
\begin{aligned}
& {\left[\begin{array}{cc}
-S_{i} & \alpha S_{i} R \\
* & -\alpha R
\end{array}\right]<0 \Longrightarrow S_{i} \alpha R S_{i}-S_{i}<0 \Longrightarrow \alpha R<S_{i}^{-1},} \\
& {\left[\begin{array}{cc}
-\beta R & I \\
* & -S_{i}
\end{array}\right]<0 \Longrightarrow S_{i}^{-1}-\beta R<0 \Longrightarrow S_{i}^{-1}<\beta R}
\end{aligned}
$$

then since $P_{i}=S_{i}^{-1}$, it arrives

$$
\alpha R<P_{i}<\beta R \Longrightarrow \alpha I<R^{-1 / 2} P_{i} R^{-1 / 2}<\beta I \Longrightarrow \alpha I<Q_{i}<\beta I
$$

and from $(6 c)$ we have

$$
\beta \gamma^{M} \delta^{2}-\alpha \varepsilon^{2}<0 \Longrightarrow \frac{\beta}{\alpha}<\frac{\varepsilon^{2}}{\gamma^{M} \delta^{2}}
$$

with both (20) and (21) we can obtain

$$
\frac{\beta}{\alpha}<\frac{\varepsilon^{2}}{\gamma^{M} \delta^{2}} \Longrightarrow \frac{\sup _{i \in \mathscr{I}}\left\{\lambda_{\max }\left(Q_{i}\right)\right\}}{\inf _{i \in \mathscr{I}}\left\{\lambda_{\min }\left(Q_{i}\right)\right\}} \leq \frac{\beta}{\alpha}<\frac{\varepsilon^{2}}{\gamma^{M} \delta^{2}},
$$

which implies (18) is satisfied. Therefore we can conclude that closed-loop switched system $((5 a),(5 b))$ is finite-time stable. Then the proof is completed.

It is worth mentioning that the class of switching signal is not specified during above discussion; thus Theorem 4 supplies us with a sufficient condition for finite-time stability of switched system ((5a), (5b)) under arbitrarily fast switching. We note that the result in Theorem 4 depends on the parameter $\gamma$. For a fixed $\gamma$, conditions (6a), (6b), and (6c) can be expressed in an LMI form. Obviously, in order to find a suitable $\gamma$, a one parameter search may be necessary; nevertheless this does not represent a hard computational problem.

Remark 5. Once the state bound $\varepsilon$ is not ascertained, a set of optimized controller gains $K_{i}=X_{i} S_{i}^{-1}$ and $K_{i, j}=X_{i, j} S_{i}^{-1}$ with minimum value $\varepsilon_{\mathrm{min}}$ is of interest. Since from (6c) we have $\varepsilon^{2}>\beta \gamma^{M} \delta^{2} / \alpha$, the minimum value $\varepsilon_{\min }$ can be found by solving optimization problem $\min \beta \gamma^{M} \delta^{2} / \alpha$ subject to (6a) and (6b). Furthermore, when $\delta$ is fixed and letting $\alpha=1$, $\beta=\kappa \alpha$ be derived through optimization procedure according to

$$
\begin{array}{ll}
\min _{\kappa \geq 1} & \kappa \\
\text { s.t. } & {\left[\begin{array}{cc}
-S_{i} & S_{i} R \\
* & -R
\end{array}\right]<0} \\
& {\left[\begin{array}{cc}
-\kappa R & I \\
* & -S_{i}
\end{array}\right]<0} \\
& {\left[\begin{array}{cc}
S_{j} & A_{i} S_{i}+B_{i} X_{i, j} \\
* & -\gamma S_{i}
\end{array}\right]<0}
\end{array}
$$

with a fixed parameter $\gamma$ and the minimum $\varepsilon_{\min }=\sqrt{\kappa \gamma^{M} \delta^{2}}$, which can be implemented on some numerical optimization software tools such as the optimization toolbox of Matlab to ascertain the optimized value $\kappa$ and finally ascertain the minimum $\varepsilon_{\min }=\sqrt{\kappa \gamma^{M} \delta^{2}}$. And when the admissible initial state bound $\delta$ is not ascertained, a set of optimized controller gains with the maximum $\delta_{\max }$ with a fixed $\varepsilon$ is of interest, and the search for $\delta_{\max }$ can be executed by a similar way.

When we let $K_{i, j}=K_{i}, \forall(i, j) \in \mathscr{I} \times \mathscr{I}$, the following design results can be derived.

Corollary 6. The closed-loop switched system $((5 a),(5 b))$ is finite-time stable with respect to $(\delta, \varepsilon, R, M)$, if there exist a set of matrices $S_{i}, X_{i}, i \in \mathscr{I}$, and positive scalars $\alpha>0, \beta>0$, and $\gamma \geq 1$ such that the following conditions are satisfied $\forall(i, j) \in$ $\mathscr{I} \times \mathscr{I}:$

$$
\begin{gathered}
{\left[\begin{array}{cc}
-S_{j} & A_{i} S_{i}+B_{i} X_{i} \\
* & -\gamma S_{i}
\end{array}\right]<0,} \\
{\left[\begin{array}{cc}
-S_{i} & \alpha S_{i} R \\
* & -\alpha R
\end{array}\right]<0, \quad\left[\begin{array}{cc}
-\beta R & I \\
* & -S_{i}
\end{array}\right]<0,} \\
\beta \gamma^{M} \delta^{2}-\alpha \varepsilon^{2}<0 .
\end{gathered}
$$

Then the set of state feedback controllers is given by $K_{i}=X_{i} S_{i}^{-1}$.

Furthermore, if we chose $S=S_{i}, \forall i \in \mathscr{I}$, then we get the following results based on search for a single positive matrix.

Corollary 7. The closed-loop switched system ((5a), (5b)) is finite-time stable with respect to $(\delta, \varepsilon, R, M)$, if there exist a set of matrices $S, X_{i}, i \in \mathscr{I}$, and positive scalars $\alpha>0$, $\beta>0$, and $\gamma \geq 1$ such that the following conditions are satisfied $\forall(i, j) \in \mathscr{I} \times \mathscr{I}$ :

$$
\begin{gathered}
{\left[\begin{array}{cc}
-S & A_{i} S+B_{i} X_{i} \\
* & -\gamma S
\end{array}\right]<0,} \\
{\left[\begin{array}{cr}
-S & \alpha S R \\
* & -\alpha R
\end{array}\right]<0, \quad\left[\begin{array}{cc}
-\beta R & I \\
* & -S
\end{array}\right]<0,} \\
\beta \gamma^{M} \delta^{2}-\alpha \varepsilon^{2}<0 .
\end{gathered}
$$

Then the set of state feedback controllers is given by $K_{i}=X_{i} S^{-1}$.

Obviously, the results in both Theorem 4 and Corollary 6 are based on searching for a set of positive matrices, which would be explicitly less conservative than that based on searching for a common positive matrix in Corollary 7. Moreover, the main advantage of Theorem 4 is that the controller for switched system ((1a), (1b)) designs a reset controller $K_{i, j}=X_{i, j} S_{i}^{-1}$ at switching instant, which could reduce the conservativeness compared to Corollary 6 only considering controller for each subsystem. To compare the conservativeness of the above proposed results, a numerical simulation is given in following example. 


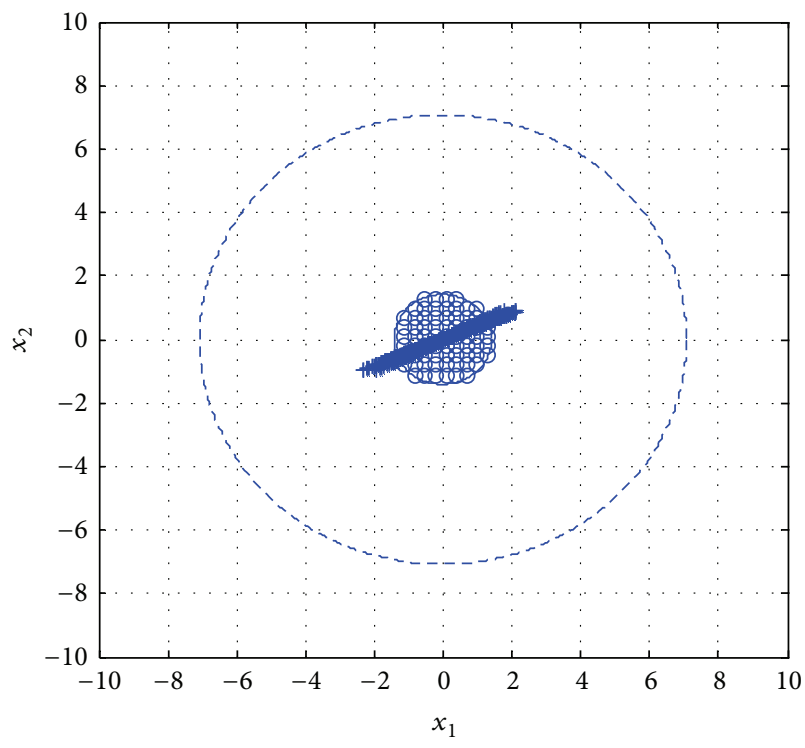

FIGURE 1: State response and minimum bound by Corollary 7.

Example 8. Consider a second order switched system with two subsystems given by

$$
\begin{array}{rlr}
A_{1} & =\left[\begin{array}{ll}
-0.0755 & -2.0776 \\
-0.3586 & -0.1435
\end{array}\right], & B_{1}=\left[\begin{array}{c}
0.2490 \\
-0.3835
\end{array}\right] ; \\
A_{2}=\left[\begin{array}{ll}
1.3933 & -0.3771 \\
0.6518 & -0.6614
\end{array}\right], & B_{2}=\left[\begin{array}{c}
-0.5285 \\
0.0554
\end{array}\right] .
\end{array}
$$

We chose $\delta=\sqrt{2}, R=I$, and $M=10$. Then we assume that $\varepsilon$ is not ascertained; our aim in this example is to design the controller ensuring the minimum value $\varepsilon_{\text {min }}$ through optimization procedure (23). In order to illustrate the advantages of our approach based on searching for a set of positive matrices and applying resetting controller at switching instant, three approaches are used.

(1) Firstly we design controller based on searching for a single positive matrix by Corollary 7; the controllers are

$$
K_{1}=\left[\begin{array}{ll}
-0.6624 & 1.4767
\end{array}\right] ; \quad K_{2}=\left[\begin{array}{ll}
0.1397 & 1.4823
\end{array}\right]
$$

and the minimum value $\varepsilon_{\min }=7.0739$. The simulation result under a random generated switching signal is given in Figure 1. Note that "o" denotes the initial state $x(0)$ and "+" denotes the discrete state $x(k), \forall k \in\{1,2, \ldots, 10\}$. The solid line denotes the bound for initial state $x(0)$ and the dotted line is the bound for discrete state $x(k), \forall k \in\{1,2, \ldots, 10\}$.

(2) Then we propose the simulation results by Corollary 6 based on searching for a set of positive matrices but without resetting controller. In this case, we only design controller for each subsystem. The controllers are

$$
K_{1}=\left[\begin{array}{ll}
-0.6691 & 1.4980
\end{array}\right] ; \quad K_{2}=\left[\begin{array}{ll}
0.8290 & 0.8676
\end{array}\right] .
$$

The minimum value $\varepsilon_{\min }=3.8430$. The simulation result is given in Figure 2.

(3) Finally we use the proposed approach by Theorem 4 based on searching for a set of positive matrices and with

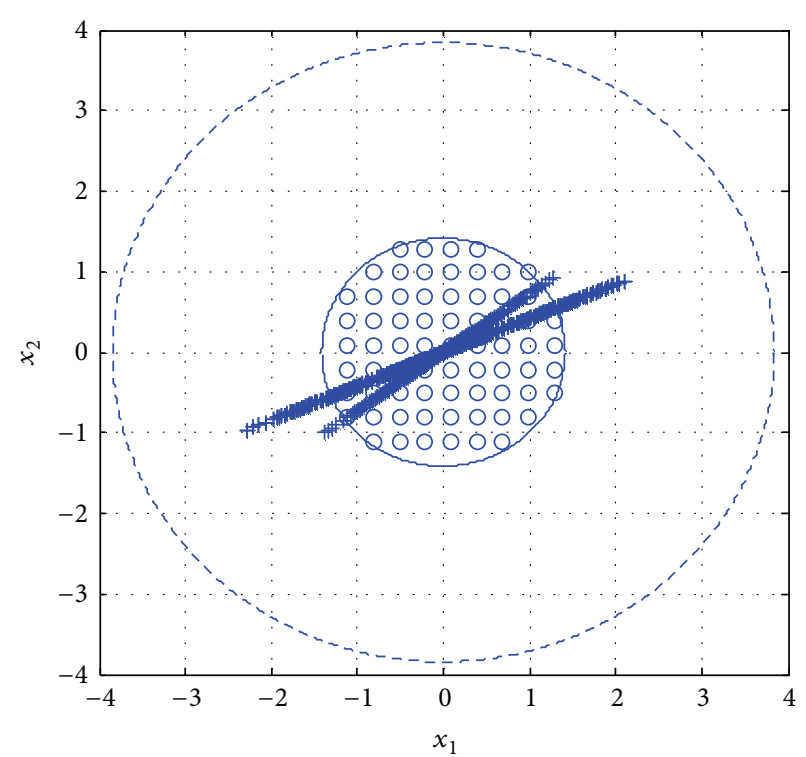

FIGURE 2: State response and minimum bound without reset controller.

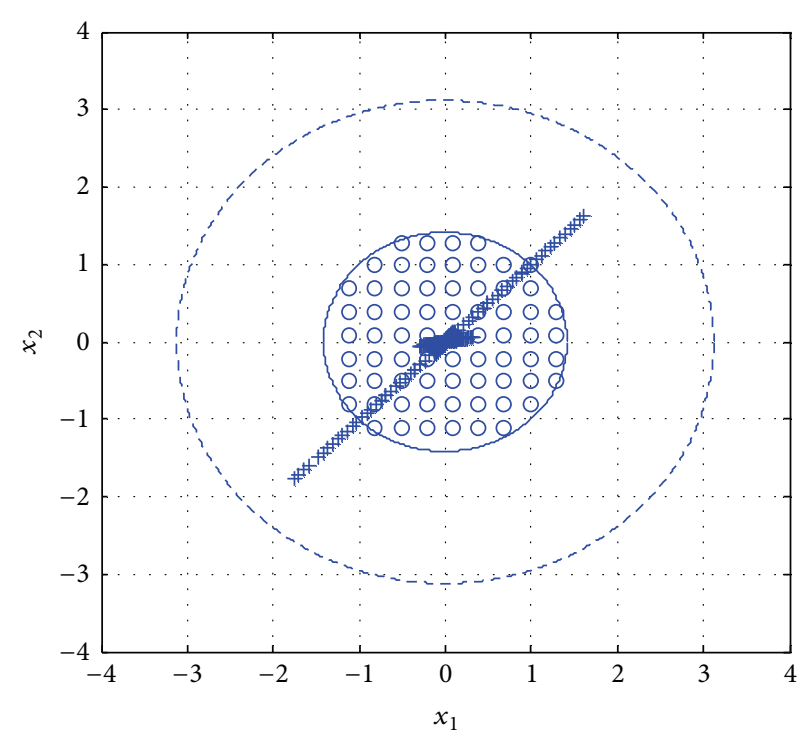

FIgURE 3: State response and minimum bound by Theorem 4 .

resetting controller. According to Theorem 4, the controllers are given by

$$
\begin{aligned}
& K_{1}=\left[\begin{array}{ll}
-0.8021 & 0.5615
\end{array}\right] ; \quad K_{2}=\left[\begin{array}{ll}
1.7337 & 0.0795
\end{array}\right], \\
& K_{1,2}=\left[\begin{array}{ll}
-0.4458 & 3.0715
\end{array}\right] ; \quad K_{2,1}=\left[\begin{array}{ll}
1.5990 & 0.1978
\end{array}\right] \text {. }
\end{aligned}
$$

The minimum value $\varepsilon_{\min }=3.1179$ which explicitly has the smallest value among the three proposed approaches. The simulation result is given in Figure 3.

Comparing three simulation results in this example, we can see that the conservativeness can be reduced by searching for a set of positive matrices and further considering the resetting controller design. 
Then we consider the static output feedback controller $u(k)=K_{i} y(k), \forall k \in \mathbb{Z}^{+} \backslash \mathcal{S}$, and $u(k)=K_{i, j} y(k), \forall k \in \mathcal{S}$ and $i \neq j,(i, j) \in \mathscr{I} \times \mathscr{I}$; the closed-loop switched system becomes

$x(k+1)=\left(A_{i}+B_{i} K_{i} C_{i}\right) x(k), \quad x(0)=x_{0}, \forall k \in \mathbb{Z}^{+} \backslash \mathcal{\delta}$,

$$
x(k+1)=\left(A_{i}+B_{i} K_{i, j} C_{i}\right) x(k), \quad \forall k \in \mathcal{S} .
$$

Motivated by the same idea, the output feedback finitetime stabilization will be investigated. At first, the following assumption is given.

Assumption 9. Matrices $C_{i}, \forall i \in \mathscr{I}$, are full of row rank.

Similar to state feedback controller design and based on Assumption 9, the following result can be derived.

Theorem 10. The closed-loop switched system ((30a), (30b)) under Assumption 9 is finite-time stable with respect to $(\delta, \varepsilon, R, M)$, if there exist a set of matrices $S_{i}, i \in \mathscr{F}, X_{i, j}$, $(i, j) \in \mathscr{I} \times \mathscr{I}$, and positive scalars $\alpha>0, \beta>0$, and $\gamma \geq 1$ such that the following conditions are satisfied $\forall(i, j) \in \mathscr{I} \times \mathscr{I}$ :

$$
\begin{gathered}
{\left[\begin{array}{cc}
-S_{j} & A_{i} S_{i}+B_{i} X_{i, j} C_{i} \\
* & -\gamma S_{i}
\end{array}\right]<0,} \\
{\left[\begin{array}{cc}
-S_{i} & \alpha S_{i} R \\
* & -\alpha R
\end{array}\right]<0, \quad\left[\begin{array}{cc}
-\beta R & I \\
* & -S_{i}
\end{array}\right]<0,} \\
\beta \gamma^{M} \delta^{2}-\alpha \varepsilon^{2}<0, \\
Y_{i} C_{i}=C_{i} S_{i} .
\end{gathered}
$$

Then the set of state feedback controllers is given by $K_{i}=$ $X_{i, i} Y_{i}^{-1}$ and $K_{i, j}=X_{i, j} Y_{i}^{-1}$.

Proof. From (31a) we know that $S_{i}, \forall i \in \mathscr{I}$, are positive matrices, and $C_{i}, \forall i \in \mathscr{I}$, are full of row rank by Assumption 9; it follows that $Y_{i}, \forall i \in \mathscr{I}$, is full of rank and invertible. Thus by (31d), letting $K_{i, i}=K_{i}$ and substituting $X_{i, j}=K_{i, j} Y_{i}$ we obtain

$$
X_{i, j} C_{i}=K_{i, j} C_{i} S_{i} .
$$

So (31a) is equivalent to

$$
\left[\begin{array}{cc}
-S_{j} & A_{i} S_{i}+B_{i} K_{i, j} C_{i} S_{i} \\
* & -\gamma S_{i}
\end{array}\right]<0 .
$$

For pre- and postmultiplying (33) by the symmetric matrix $\left[\begin{array}{cc}S_{j}^{-1} & 0 \\ * & S_{i}^{-1}\end{array}\right]$, the following equivalent condition of (33) is obtained as

$$
\left[\begin{array}{cc}
-S_{j}^{-1} & S_{j}^{-1}\left(A_{i}+B_{i} K_{i, j} C_{i}\right) \\
* & -\gamma S_{i}^{-1}
\end{array}\right]<0 .
$$

Let $P_{j}=S_{j}^{-1}$ and $P_{i}=S_{i}^{-1}$ in (34); we have

$$
\left[\begin{array}{cc}
-P_{j} & P_{j}\left(A_{i}+B_{i} K_{i, j} C_{i}\right) \\
* & -\gamma P_{i}
\end{array}\right]<0 .
$$

Then by Lemma 2 we get $\forall(i, j) \in \mathscr{I} \times \mathscr{I}$

$$
\begin{aligned}
& {\left[\begin{array}{cc}
-P_{j} & P_{j}\left(A_{i}+B_{i} K_{i, j} C_{i}\right) \\
* & -\gamma P_{i}
\end{array}\right]<0} \\
& \Longrightarrow\left(A_{i}+B_{i} K_{i, j} C_{i}\right)^{T} P_{j}\left(A_{i}+B_{i} K_{i, j} C_{i}\right)-\gamma P_{i}<0 .
\end{aligned}
$$

Let $V_{i}(k)=x^{T}(k) P_{i} x(k), i \in \mathscr{I}$ for each subsystem of closed-loop ((30a), (30b)); then we construct the following Lyapunov-like function:

$$
V(k)=\sum_{i \in \mathscr{I}} \theta_{i}(k) V_{i}(k)=\sum_{i \in \mathscr{I}} x^{T}(k) \theta_{i}(k) P_{i} x(k),
$$

where $\theta_{i}(\cdot): \mathbb{Z}^{+} \rightarrow\{0,1\}$ and $\sum_{i \in \mathscr{I}} \theta_{i}(k)=1$. Thus, (36) implies that

$$
V(k+1)<\gamma V(k) .
$$

Then from (31b) and (31c) and by the same guideline in Theorem 4, we can establish the finite-time stability of closedloop switched system ((30a), (30b)). Then the proof is completed.

Remark 11. As what is discussed in Remark 3, a set of optimized controller gains $K_{i}=X_{i, i} Y_{i}^{-1}$ and $K_{i, j}=X_{i, j} Y_{i}^{-1}$ with the minimum $\varepsilon_{\text {min }}$ can be derived through similar optimization procedure according to

$$
\begin{array}{ll}
\min _{\kappa \geq 1} & \kappa \\
\text { s.t. } & {\left[\begin{array}{cc}
-S_{i} & S_{i} R \\
* & -R
\end{array}\right]<0} \\
& {\left[\begin{array}{cc}
-\kappa R & I \\
* & -S_{i}
\end{array}\right]<0} \\
& {\left[\begin{array}{cc}
-S_{j} & A_{i} S_{i}+B_{i} X_{i, j} C_{i} \\
* & -\gamma S_{i}
\end{array}\right]<0}
\end{array}
$$

with a fixed parameter $\gamma$ and the minimum $\varepsilon_{\min }=\sqrt{\kappa \gamma^{M} \delta^{2}}$.

\section{Finite-Time Stabilization under Slow Switching}

In this section, the finite-time stabilization under slow switching is considered. Now we consider a class of average dwell time switching; the definition of average dwell time is given as follows.

Definition 12 (see [13]). For switched system ((1a), (1b)) and any $k_{2}>k_{1} \geq 0$, let $N\left(k_{1}, k_{2}\right)$ be the number of switchings in the interval $\left[k_{1}, k_{2}\right)$. If for any given positive numbers $N_{0} \geq 0$ and $\tau_{a}>0$ we have $N\left(k_{2}, k_{1}\right) \leq N_{0}+\left(k_{2}-k_{1}\right) / \tau_{a}$, then $\tau_{a}$ and $N_{0}$ are called average dwell time and chatter bound.

The following closed-loop switched system is considered: $x(k+1)=\left(A_{i}+B_{i} K_{i}\right) x(k), \quad x(0)=x_{0}, \forall k \in \mathbb{Z}^{+}$. 
Thus, the objective here is to design controller $u(k)=K_{i} x(k)$, $i \in \mathscr{I}$, ensuring the finite-time stability of closed-loop switched system ((1a), (1b)) with the admissible average dwell time.

Theorem 13. The closed-loop switched system (40) is finitetime stable with respect to $(\delta, \varepsilon, R, M)$, if there exist a set of matrices $S_{i}, X_{i}, i \in \mathcal{I}$, and positive scalars $\alpha>0, \beta>0$, and $\gamma \geq 1$ such that the following conditions are satisfied

$$
\begin{gathered}
{\left[\begin{array}{cc}
-S_{i} & A_{i} S_{i}+B_{i} X_{i} \\
* & -\gamma S_{i}
\end{array}\right]<0,} \\
{\left[\begin{array}{cc}
-S_{i} & \alpha S_{i} R \\
* & -\alpha R
\end{array}\right]<0, \quad\left[\begin{array}{cc}
-\beta R & I \\
* & -S_{i}
\end{array}\right]<0,} \\
\tau_{a}>\frac{M(\ln \beta-\ln \alpha)}{\ln \varepsilon^{2}-\ln \gamma^{M} \delta^{2}-\left(N_{0}+1\right)(\ln \beta-\ln \alpha)} .
\end{gathered}
$$

Then the set of state feedback controllers is given by $K_{i}=X_{i} S_{i}^{-1}$, $i \in \mathscr{I}$.

Proof. Substituting $X_{i}=K_{i} S_{i}, i \in \mathscr{I}$, into (41a) we get

$$
\left[\begin{array}{cc}
-S_{i} & A_{i} S_{i}+B_{i} K_{i} S_{i} \\
* & -\gamma S_{i}
\end{array}\right]<0
$$

For pre- and postmultiplying (42) by the symmetric matrix $\left[\begin{array}{cc}S_{i}^{-1} & 0 \\ * & S_{i}^{-1}\end{array}\right]$, the following equivalent condition of (42) is obtained:

$$
\left[\begin{array}{cc}
-S_{i}^{-1} & S_{i}^{-1}\left(A_{i}+B_{i} K_{i}\right) \\
* & -\gamma S_{i}^{-1}
\end{array}\right]<0 .
$$

Let $P_{i}=S_{i}^{-1}$ in (43); we have

$$
\left[\begin{array}{cc}
-P_{i} & P_{i}\left(A_{i}+B_{i} K_{i}\right) \\
* & -\gamma P_{i}
\end{array}\right]<0 .
$$

Then by Lemma 2 we get

$$
\begin{aligned}
& {\left[\begin{array}{cc}
-P_{i} & P_{i}\left(A_{i}+B_{i} K_{i, j}\right) \\
* & -\gamma P_{i}
\end{array}\right]<0} \\
& \Longrightarrow\left(A_{i}+B_{i} K_{i}\right)^{T} P_{i}\left(A_{i}+B_{i} K_{i}\right)-\gamma P_{i}<0 .
\end{aligned}
$$

Let $V_{i}(k)=x^{T}(k) P_{i} x(k), i \in \mathscr{I}$, for each subsystem of closed-loop (40); then we construct following Lyapunov-like function:

$$
V(k)=\sum_{i \in \mathscr{F}} \theta_{i}(k) V_{i}(k)=\sum_{i \in \mathscr{F}} x^{T}(k) \theta_{i}(k) P_{i} x(k),
$$

where $\theta_{i}(\cdot): \mathbb{Z}^{+} \rightarrow\{0,1\}$ and $\sum_{i \in \mathcal{F}} \theta_{i}(k)=1$. And by (45) we obtain

$$
V(k+1)<\gamma V(k) .
$$

Similar to the proof line in Theorem 4, from (41b) we get

$$
\alpha R<P_{i}<\beta R, \quad \forall i \in \mathscr{I} .
$$

We can easily obtain from (47) that

$$
\begin{aligned}
V(k) & <\gamma^{\left(k-k_{l}\right)} V\left(k_{l}\right)<\frac{\beta}{\alpha} \gamma^{\left(k-k_{l-1}\right)} V\left(k_{l-1}\right) \\
& <\cdots<\left(\frac{\beta}{\alpha}\right)^{N(0, k)} \gamma^{M} V(0) .
\end{aligned}
$$

By Definition 12, we get

$$
\begin{aligned}
V(k) & <\left(\frac{\beta}{\alpha}\right)^{N(0, k)} \gamma^{M} V(0)<\left(\frac{\beta}{\alpha}\right)^{N_{0}+k / \tau_{a}} \gamma^{M} V(0) \\
& <\left(\frac{\beta}{\alpha}\right)^{N_{0}+M / \tau_{a}} \gamma^{M} V(0) .
\end{aligned}
$$

Then let $Q_{i}=R^{-1 / 2} P_{i} R^{-1 / 2}, i \in \mathscr{I}$; we get

$$
\begin{aligned}
V(k) & =x^{T}(k) P_{i} x(k) \\
& =x^{T}(k) R^{1 / 2} R^{-1 / 2} P_{i} R^{-1 / 2} R^{1 / 2} x(k) \\
& =x^{T}(k) R^{1 / 2} Q_{i} R^{1 / 2} x(k) \\
& \geq \inf _{i \in \mathscr{I}}\left\{\lambda_{\min }\left(Q_{i}\right)\right\} x^{T}(k) R x(k) .
\end{aligned}
$$

On the other hand $\forall i \in \mathscr{I}$ we have

$$
\begin{aligned}
V(0) & =x^{T}(0) P_{i} x(0) \\
& =x^{T}(0) R^{1 / 2} Q_{i} R^{1 / 2} x(0) \\
& \leq \sup _{i \in \mathscr{I}}\left\{\lambda_{\max }\left(Q_{i}\right)\right\} x^{T}(0) R x(0) ;
\end{aligned}
$$

using the fact that $x^{T}(0) R x(0) \leq \delta^{2}$ we get

$$
V(0) \leq \sup _{i \in \mathscr{I}}\left\{\lambda_{\max }\left(Q_{i}\right)\right\} \delta^{2} .
$$

Using (50), (51), and (53) altogether, the following inequality can be derived:

$$
x^{T}(k) R x(k) \leq\left(\frac{\beta}{\alpha}\right)^{N_{0}+M / \tau_{a}} \gamma^{M} \delta^{2} \frac{\sup _{i \in \mathcal{F}}\left\{\lambda_{\max }\left(Q_{i}\right)\right\}}{\inf _{i \in \mathscr{I}}\left\{\lambda_{\min }\left(Q_{i}\right)\right\}} .
$$

Now, to ensure the finite-time boundness of switched system (40) with respect to $(\delta, \varepsilon, R, M)$, the following condition should be satisfied:

$$
\frac{\sup _{i \in \mathscr{I}}\left\{\lambda_{\max }\left(Q_{i}\right)\right\}}{\inf _{i \in \mathscr{F}}\left\{\lambda_{\min }\left(Q_{i}\right)\right\}} \leq \frac{\varepsilon^{2}}{(\beta / \alpha)^{N_{0}+M / \tau_{a}} \gamma^{M} \delta^{2}} ;
$$

since we have

$\alpha R<P_{i}<\beta R \Longrightarrow \alpha I<R^{-1 / 2} P_{i} R^{-1 / 2}<\beta I \Longrightarrow \alpha I<Q_{i}<\beta I$, 


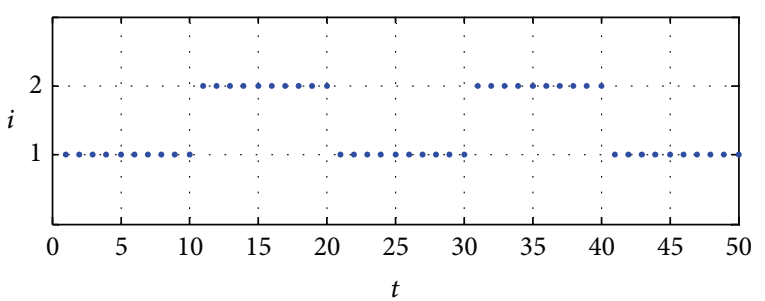

Figure 4: Periodical switching signal.

and from (41c) we have

$$
\begin{aligned}
\tau_{a}>\frac{M(\ln \beta-\ln \alpha)}{\ln \varepsilon^{2}-\ln \gamma^{M} \delta^{2}-\left(N_{0}+1\right)(\ln \beta-\ln \alpha)} \\
\Longleftrightarrow \frac{\beta}{\alpha}<\frac{\varepsilon^{2}}{(\beta / \alpha)^{N_{0}+M / \tau_{a}} \gamma^{M} \delta^{2}}
\end{aligned}
$$

both with (56) and (57) we can obtain

$$
\begin{aligned}
& \frac{\beta}{\alpha}<\frac{\varepsilon^{2}}{(\beta / \alpha)^{N_{0}+M / \tau_{a}} \gamma^{M} \delta^{2}} \\
& \Longrightarrow \frac{\sup _{i \in \mathscr{I}}\left\{\lambda_{\max }\left(Q_{i}\right)\right\}}{\inf _{i \in \mathscr{I}}\left\{\lambda_{\min }\left(Q_{i}\right)\right\}} \leq \frac{\beta}{\alpha}<\frac{\varepsilon^{2}}{(\beta / \alpha)^{N_{0}+M / \tau_{a}} \gamma^{M} \delta^{2}}
\end{aligned}
$$

Therefore we can conclude that closed-loop switched system (40) is finite-time bounded. Then the proof is completed.

Example 14. Consider switched system with 2 subsystems as follows:

$$
\begin{array}{ll}
A_{1}=\left[\begin{array}{ll}
-0.0755 & -2.0776 \\
-0.3586 & -0.1435
\end{array}\right], & B_{1}=\left[\begin{array}{c}
0.2490 \\
-0.3835
\end{array}\right] ; \\
A_{2}=\left[\begin{array}{ll}
1.3933 & -0.3771 \\
0.6518 & -0.6614
\end{array}\right], & B_{2}=\left[\begin{array}{c}
-0.5285 \\
0.0554
\end{array}\right] .
\end{array}
$$

We chose $(\delta=\sqrt{2}, \varepsilon=5, R=I, M=50)$; by Theorem 13 the state feedback controller is designed as

$$
K_{1}=\left[\begin{array}{ll}
-0.4968 & 0.2282
\end{array}\right] ; \quad K_{2}=\left[\begin{array}{ll}
-0.1628 & -0.0384
\end{array}\right]
$$

and the admissible average dwell time $\tau_{a}>9.9366$; thus we chose a periodical switching that the switching occurs every 10 seconds which is shown in Figure 4.

The simulation result is in Figure 5.

It is explicit that the controller and the switching law with the admissible average dwell time meet requirement of finitetime stabilization.

Then we consider the static output feedback controller $u(k)=K_{i} y(k), \forall k \in \mathbb{Z}^{+}$; the closed-loop switched system becomes

$$
x(k+1)=\left(A_{i}+B_{i} K_{i} C_{i}\right) x(k), \quad x(0)=x_{0}, \forall k \in \mathbb{Z}^{+} .
$$

Similar to the state feedback case, the following results can be derived.

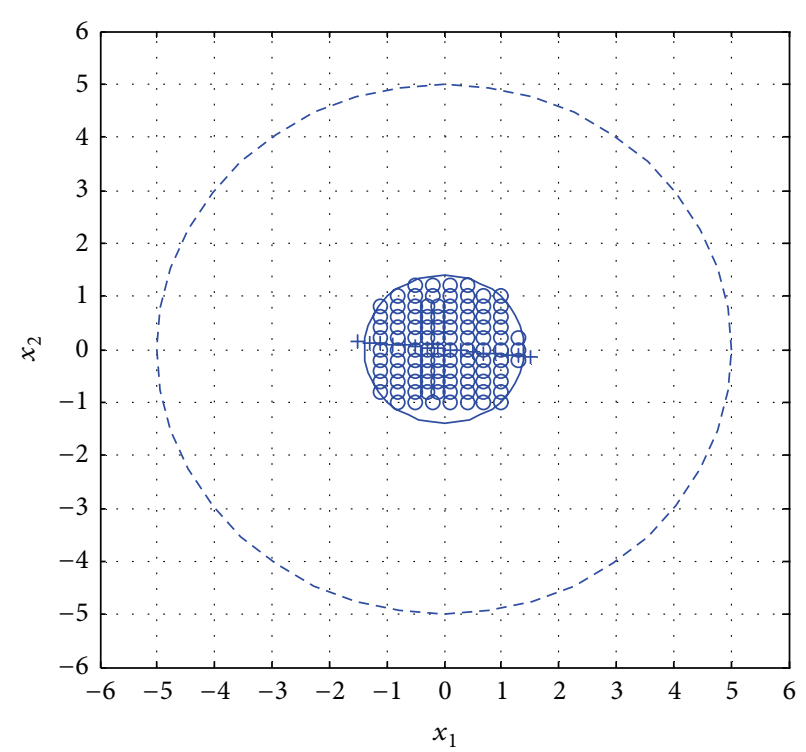

FIgURE 5: State response and minimum bound by Theorem 13.

Theorem 15. The closed-loop switched system (61) under Assumption 9 is finite-time stable with respect to $(\delta, \varepsilon, R, M)$, if there exist a set of matrices $S_{i}, X_{i}, i \in \mathscr{I}$, and positive scalars $\alpha>0, \beta>0$, and $\gamma \geq 1$ such that the following conditions are satisfied:

$$
\begin{gathered}
{\left[\begin{array}{cc}
-S_{i} & A_{i} S_{i}+B_{i} X_{i} C_{i} \\
* & -\gamma S_{i}
\end{array}\right]<0,} \\
{\left[\begin{array}{cc}
-S_{i} & \alpha S_{i} R \\
* & -\alpha R
\end{array}\right]<0, \quad\left[\begin{array}{cc}
-\beta R & I \\
* & -S_{i}
\end{array}\right]<0,} \\
\tau_{a}>\frac{M(\ln \beta-\ln \alpha)}{\ln \varepsilon^{2}-\ln \gamma^{M} \delta^{2}-\left(N_{0}+1\right)(\ln \beta-\ln \alpha)}, \\
Y_{i} C_{i}=C_{i} S_{i} .
\end{gathered}
$$

Then the set of state feedback controllers is given by $K_{i}=X_{i} Y_{i}^{-1}$.

Proof. From the similar guideline in Theorem 10, let $P_{i}=S_{i}^{-1}$, we have

$$
\left[\begin{array}{cc}
-P_{i} & P_{j}\left(A_{i}+B_{i} K_{i} C_{i}\right) \\
* & -\gamma P_{i}
\end{array}\right]<0 .
$$

Let $V_{i}(k)=x^{T}(k) P_{i} x(k), i \in \mathscr{I}$, for each subsystem of closedloop system (61); then we construct the following Lyapunovlike function:

$$
V(k)=\sum_{i \in \mathscr{I}} \theta_{i}(k) V_{i}(k)=\sum_{i \in \mathscr{I}} x^{T}(k) \theta_{i}(k) P_{i} x(k),
$$

where $\theta_{i}(\cdot): \mathbb{Z}^{+} \rightarrow\{0,1\}$ and $\sum_{i \in \mathscr{F}} \theta_{i}(k)=1$. By (63) and (64) we can easily get that

$$
V(k+1)<\gamma V(k) .
$$

Then from the same guideline in Theorem 13, (62b) and (62c) guarantee the finite-time stability of closed-loop switched system (61). 


\section{Conclusions}

The problem of finite-time stabilization for switched discretetime system is investigated in this paper. Combining the controllers for subsystems and resetting controller at switching instant, the state and output feedback controllers are designed under fast switching. The controller can be obtained by solving a set of LMIs. Then under slow switching, the state and output feedback controllers for subsystems are designed with admissible average dwell time. Several numerical simulations are given to illustrate our approach in this paper.

\section{Conflict of Interests}

The authors (Liaoliang Xiao and Mingyang $\mathrm{Yu}$ ) declare that there is no conflict of interests regarding the publication of this paper.

\section{Acknowledgment}

This work is supported by Hunan Provincial Education Department (no. 13C592).

\section{References}

[1] D. Liberzon, Switching in Systems and Control, Systems \& Control: Foundations \& Applications, Birkhäuser, Boston, Mass, USA, 2003.

[2] Z. Sun and S. S. Ge, Switched Linear Systems-Control and Design, Springer, London, UK, 2005.

[3] R. A. Decarlo, M. S. Branicky, S. Pettersson, and B. Lennartson, "Perspectives and results on the stability and stabilizability of hybrid systems," Proceedings of the IEEE, vol. 88, no. 7, pp. 10691082, 2000.

[4] A. Balluchi, M. D. Benedetto, C. Pinello, C. Rossi, and A. Sangiovanni-Vincentelli, "Cut-off in engine control: a hybrid system approach," in Proceedings of the 36th IEEE Conference on Decision and Control, pp. 4720-4725, 1997.

[5] B. E. Bishop and M. W. Spong, "Control of redundant manipulators using logic-based switching," in Proceedings of the 36th IEEE Conference on Decision and Control, pp. 16-18, 1998.

[6] W. Zhang, M. S. Branicky, and S. M. Phillips, "Stability of networked control systems," IEEE Control Systems Magazine, vol. 21, no. 1, pp. 84-97, 2001.

[7] D. Liberzon and A. S. Morse, "Basic problems in stability and design of switched systems," IEEE Control Systems Magazine, vol. 19, no. 5, pp. 59-70, 1999.

[8] H. Lin and P. J. Antsaklis, "Stability and stabilizability of switched linear systems: a survey of recent results," IEEE Transactions on Automatic Control, vol. 54, no. 2, pp. 308-322, 2009.

[9] K. S. Narendra and J. Balakrishnan, "A common Lyapunov function for stable LTI systems with commuting A-matrices," IEEE Transactions on Automatic Control, vol. 39, no. 12, pp. 2469-2471, 1994.

[10] M. S. Branicky, "Multiple Lyapunov functions and other analysis tools for switched and hybrid systems," IEEE Transactions on Automatic Control, vol. 43, no. 4, pp. 475-482, 1998.

[11] W. Xiang, J. Xiao, and M. N. Iqbal, "Asymptotic stability, $l_{2}$ gain, boundness analysis, and control synthesis for switched systems: a switching frequency approach," International Journal of Adaptive Control and Signal Processing, vol. 26, no. 4, pp. 350373, 2012.

[12] W. Xiang and J. Xiao, "Stabilization of switched continuoustime systems with all modes unstable via dwell time switching," Automatica, vol. 50, no. 3, pp. 940-945, 2014.

[13] J. P. Hespanha, D. Liberzon, and A. S. Morse, "Stability of switched systems with average dwell-time," in Proceedings of the 38th IEEE Conference on Decision and Control (CDC '99), pp. 2655-2660, December 1999.

[14] W. Xiang and J. Xiao, "Convex sufficient conditions on asymptotic stability and $l_{2}$ gain performance for uncertain discretetime switched linear systems," IET Control Theory \& Applications, vol. 8, no. 3, pp. 211-218, 2014.

[15] L. Weiss and E. F. Infante, "Finite time stability under perturbing forces and on product spaces," IEEE Transactions on Automatic Control, vol. 12, pp. 54-59, 1967.

[16] A. N. Michel and S. H. Wu, "Stability of discrete systems over a finite interval of time," International Journal of Control, vol. 9, pp. 679-693, 1969.

[17] H. DAngelo, Linear Time-Varying Systems: Analysis and Synthesis, Allyn and Bacon, Boston, Mass, USA, 1970.

[18] F. Amato, M. Ariola, and C. Cosentino, "Finite-time stabilization via dynamic output feedback," Automatica, vol. 42, no. 2, pp. 337-342, 2006.

[19] F. Amato and M. Ariola, "Finite-time control of discrete-time linear systems," IEEE Transactions on Automatic Control, vol. 50, no. 5, pp. 724-729, 2005.

[20] W. Xiang and J. Xiao, " $H_{\infty}$ finite-time control for switched nonlinear discrete-time systems with norm-bounded disturbance," Journal of the Franklin Institute. Engineering and Applied Mathematics, vol. 348, no. 2, pp. 331-352, 2011.

[21] W. Xiang and J. Xiao, "Finite-time stability analysis and stabilization for switched linear systems," International Journal of System Science, vol. 44, no. 2, pp. 384-400, 2013.

[22] S. Boyd, L. El Ghaoui, E. Feron, and V. Balakrishnan, Linear Matrix Inequalities in System and Control Theory, vol. 15 of SIAM Studies in Applied Mathematics, Society for Industrial and Applied Mathematics (SIAM), Philadelphia, Pa, USA, 1994. 


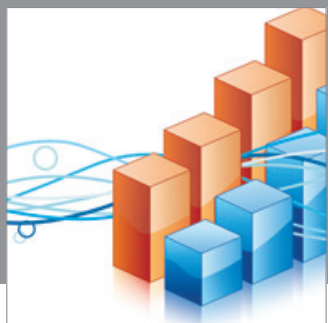

Advances in

Operations Research

mansans

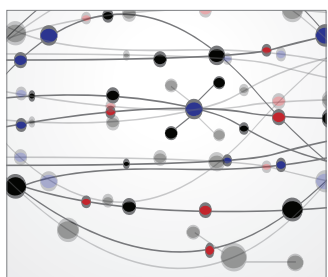

The Scientific World Journal
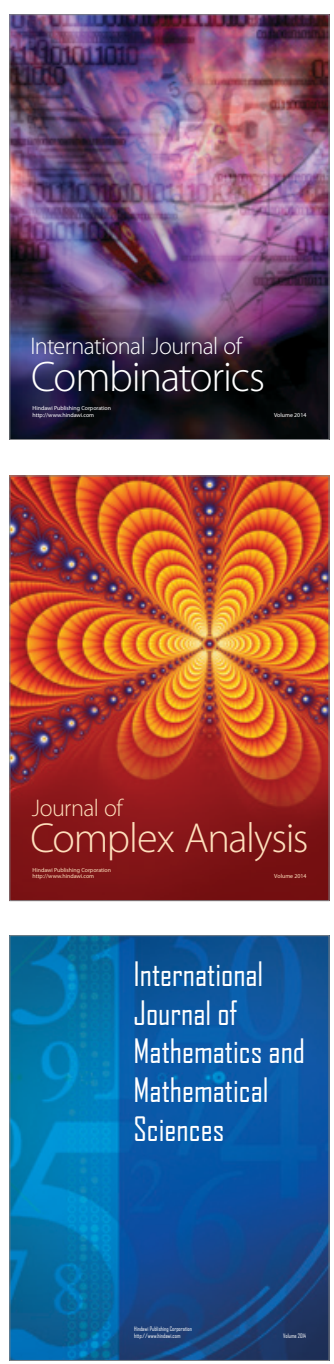
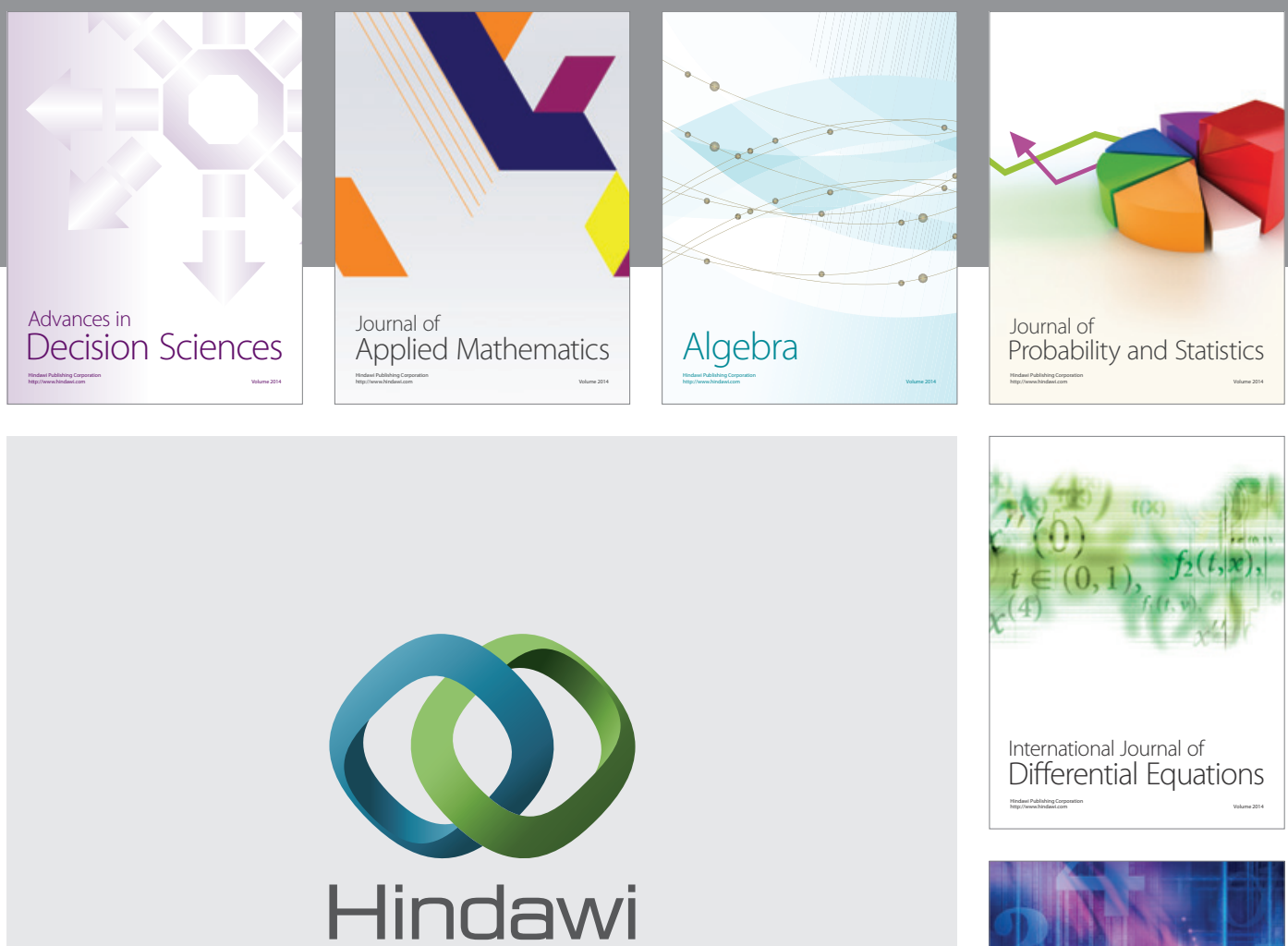

Submit your manuscripts at http://www.hindawi.com
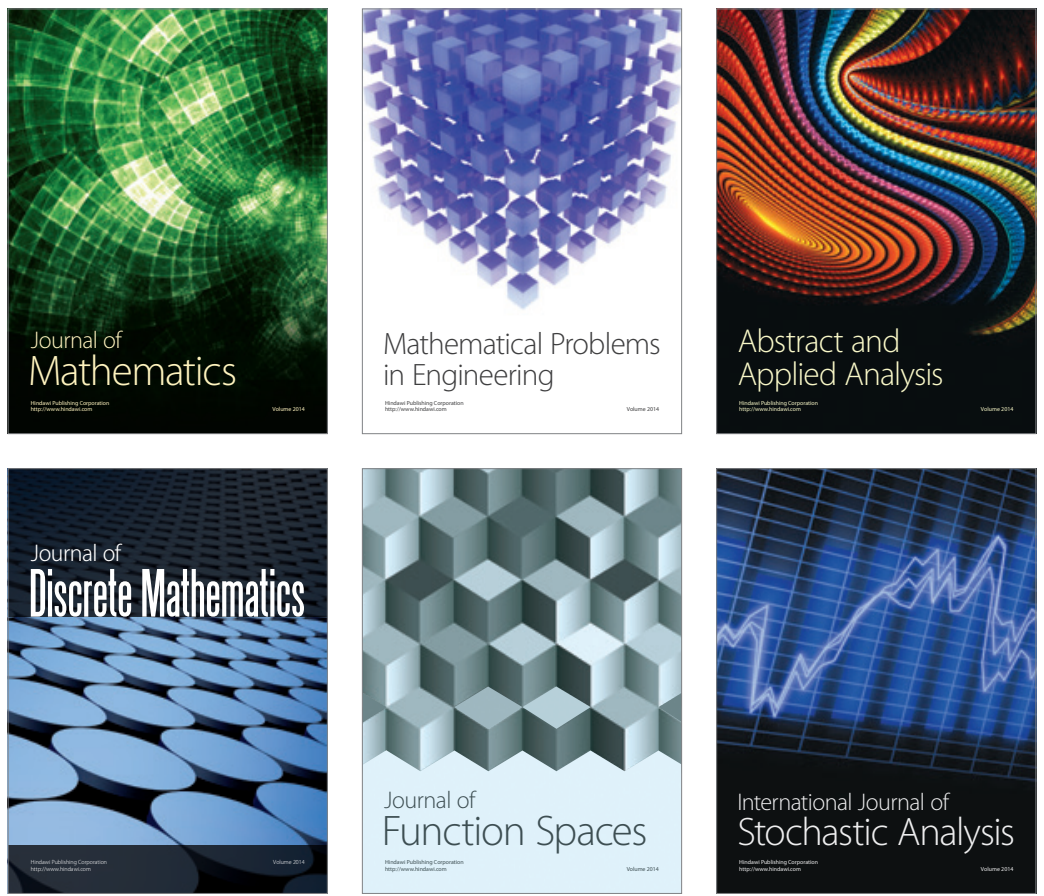

Journal of

Function Spaces

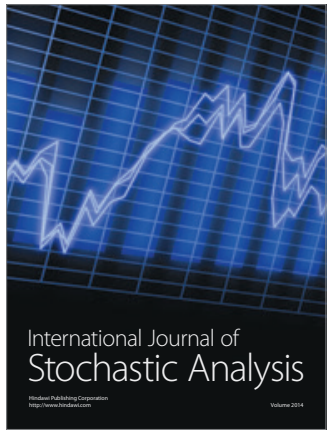

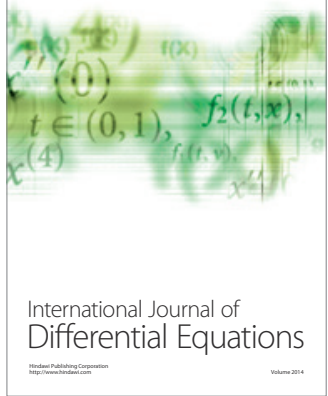
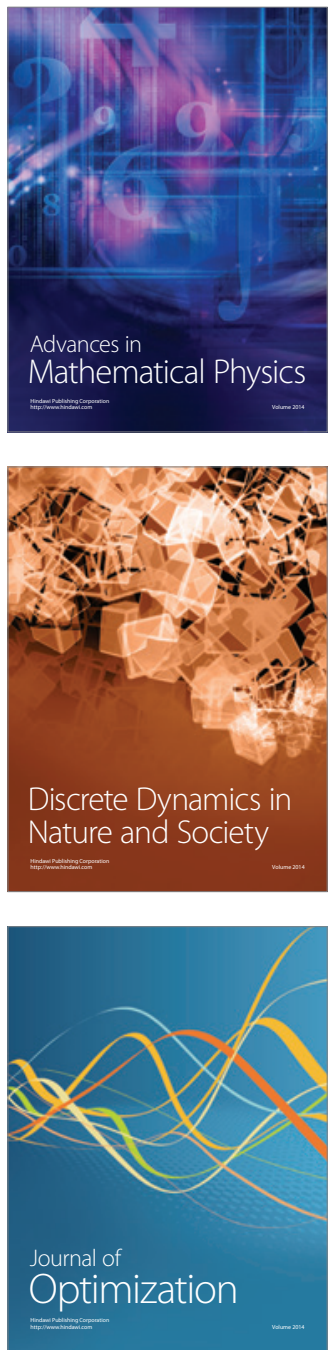